\title{
Reproducing fractional monomials: weakening of the Strang-Fix conditions
}

\author{
Victor G. Zakharov \\ victor@icmm.ru
}

July 28,2020

\begin{abstract}
A method to reproduce causal and symmetric monomials of fractional degree by integer shifts of the corresponding fractional B-splines, introduced by M. Unser and Th. Blue, is presented. Thus the traditional relation between the degree of reproduced monomials and the order of approximation holds. Bivariate, obtained by tensor product, fractional B-splines are introduced; and reproducing of bivariate causal and symmetric monomials is shown. Demonstration that the method is based on a weakening of the Strang-Fix conditions is presented.

Keywords: Fractional B-splines; causal and symmetric monomials; monomials with fractional exponents; Strang-Fix conditions
\end{abstract}

2020 MSC: 41A15, 41A30, 41A63, 65D07, 26A33

\section{Introduction}

Recall that the famous Schoenberg cardinal polynomial B-splines, see [5], are formed by peace-wise algebraic polynomials joining with the maximal continuity; and the splines can be defined as follows

$$
B^{n}(x):=\int_{\mathbb{R}} B^{n-1}(x-y) B^{0}(y) d y, \quad \text { where } \quad B^{0}(x):= \begin{cases}1, & x \in[0,1), \\ 0, & \text { otherwise. }\end{cases}
$$

Remark 1.1. In the paper, in contrast to the traditional manner, we prefer to denote Bsplines such that the order of a spline coincides with the degree. By the by, M. Unser and Th. Blue [7, 8] used similar method to define the fractional B-splines.

This is well known that the Fourier transform of any monomial $x^{n}, x \in \mathbb{R}, n \in \mathbb{Z}_{\geq 0}$, is the $n$-th derivative of the Dirac delta-distribution; and this simple relation between a monomial and its Fourier transform cannot be extended to a fractional degree. Nevertheless, the fashion of the Fourier transform of causal

$$
x_{+}^{\alpha}:=\left\{\begin{array}{ll}
x^{\alpha}, & x \geq 0, \\
0, & \text { otherwise }
\end{array} \quad \text { and symmetric } \quad x_{*}^{\alpha}:= \begin{cases}|x|^{\alpha}, & \alpha \notin 2 \mathbb{Z}_{\geq 0}, \\
x^{\alpha} \log |x|, & \alpha \in 2 \mathbb{Z}_{\geq 0}\end{cases}\right.
$$

monomials is the same for any fractional degree $\alpha \in \mathbb{R}, a>-1$, 3]. Degree-independence of the Fourier transform form of the causal and symmetric monomials allowed M. Unser and Th. Blue to extend the B-splines to non-integral orders $\alpha \in R, \alpha>-1$. Such non-integral B-splines we call "fractional". 
M. Unser and Th. Blue [7, 8, demonstrated that the fractional (causal and symmetric) B-splines of order $\alpha$ satisfy the Strang-Fix conditions [2, 6] of integral order $\lceil\alpha\rceil$ and consequently reproduce algebraic polynomials up to integral degree $\lceil\alpha\rceil$. However, Unser and Blue [8] proved that a fractional B-spline of order $\alpha$ has the fractional order of approximation $\alpha+1$.

In the paper, we state a method to reproduce fractional monomials $x_{+}^{\alpha}, x_{*}^{\alpha}$ by integer shifts of the corresponding fractional B-splines. Thus we have more traditional situation, namely, for a B-spline of non-integral order $\alpha$, we have that the degree of the reproduced monomial is fractional value $\alpha$ and the order of approximation of $B^{\alpha}$ is also fractional value $\alpha+1$. Moreover, we can note that, for the causal B-splines and causal monomials, like the classical Strang-Fix theory, we have exact reproducing by a finite number (for any bounded interval of the ray $[0, \infty)$ ) of the shifted B-splines. On the other hand, since the fractional B-splines are not compactly supported, the reproducing of the ordinary monomials $x^{n}$, $n=0,1, \ldots,\lceil\alpha\rceil$, by the fractional B-splines is not exact and converges non-uniformly (only point-wise). Note also, in spite of the infinite support of symmetric B-splines, the symmetric (fractional) monomials are reproduced by the symmetric B-splines uniformly.

Using tensor product, bivariate causal and symmetric (fractional, in general) B-splines are presented. And reproducing of the corresponding (causal and symmetric) monomials is demonstrated. Similarly to the one-dimensional case, the bivariate causal monomials are reproduced exactly (for any bounded set of the first quadrant of the plane $\mathbb{R}^{2}$ ) by a finite number of the bivariate causal B-splines.

To reproduce fractional monomials, we introduce a generalization (in fact, an weakening) of the Strang-Fix conditions.

The paper is organized as follows. In Sec. 2, we present known, and introduced by Unser and Blue [7, 8, notations, definitions, and formulas. In particular, the binomial series and the Fourier transforms for the causal and symmetric cases are demonstrated. Sec. 3 is devoted to fractional causal and symmetric B-splines and is based on the papers [7, 8] of M. Unser, Th. Blue. In Subsec. 3.3. we concern shortly that the classical (integral) Bsplines can be considered as causal and symmetric splines. In Sec. 4, we present the method to reproduce fractional, generally, causal and symmetric monomials by integer shifts of the corresponding B-splines. In Sec. 5, we consider reproducing of causal and symmetric monomials from the Strang-Fix conditions point of view. In particular, in Subsec. 5.2, a weakening of the Strang-Fix conditions is shown.

\section{Notations and definitions}

\subsection{General notations}

Here we introduce some well-known notations and definitions.

Let $\delta_{n 0}:=\left\{\begin{array}{ll}1, & n=0, \\ 0, & n \neq 0\end{array}\right.$ be the Kronecker delta and $\delta$ be the Dirac delta-distribution. Let $S^{\prime}$ denote a space of tempered distributions.

Remark 2.1. In the paper, we shall denote numerical sequences by bold symbols and elements of the sequences by the corresponding plain symbols: $\boldsymbol{p}:=\left(p_{n}\right)_{n \in \mathbb{Z}}$.

The discrete-domain Fourier transform (DDFT) $\mathfrak{F}: \mathbb{Z} \rightarrow[0,2 \pi)$ is defined as follows

$$
\mathfrak{F}[\boldsymbol{p}](\omega):=\sum_{n \in \mathbb{Z}} p_{n} e^{-i n \omega}=: \hat{\boldsymbol{p}}(\omega), \quad \omega \in[0,2 \pi) .
$$


The inverse DDFT $\mathfrak{F}^{-1}:[0,2 \pi) \rightarrow \mathbb{Z}$ is defined as

$$
p_{n}=\int_{[0,2 \pi)} \hat{\boldsymbol{p}}(\omega) e^{i n \omega} d \omega, \quad n \in \mathbb{Z}
$$

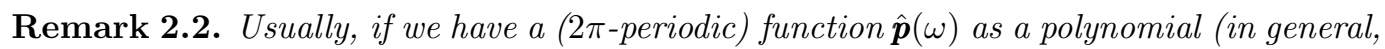
the polynomial can have terms with negative degree) or series (the Laurent series, generally) in $e^{-i \omega}$, then we can take the coefficients of the polynomial (series) $\hat{\boldsymbol{p}}$ as elements of the corresponding sequence $\boldsymbol{p}$ without evaluation of the integral in formula (2.1).

The discrete convolution $\boldsymbol{p} * \boldsymbol{q}$ of two sequences $\boldsymbol{p}:=\left(p_{n}\right)_{n \in \mathbb{Z}}, \boldsymbol{q}:=\left(q_{n}\right)_{n \in \mathbb{Z}}$ is defined as usually

$$
(\boldsymbol{p} * \boldsymbol{q})_{n}:=\sum_{k \in \mathbb{Z}} p_{n-k} q_{k}, \quad n \in \mathbb{Z} .
$$

Recall an important property of the DDFT:

$$
\mathfrak{F}[\boldsymbol{p} * \boldsymbol{q}](\omega)=\hat{\boldsymbol{p}}(\omega) \hat{\boldsymbol{q}}(\omega) .
$$

By $\boldsymbol{p} \cdot \boldsymbol{q}$ denote the inner product: $\boldsymbol{p} \cdot \boldsymbol{q}:=\sum_{k \in \mathbb{Z}} p_{k} q_{k}$.

The continuous Fourier transform (CFT) $F: \mathbb{R} \rightarrow \widehat{\mathbb{R}}$ of a function $f \in L^{1}(\mathbb{R})$ is defined as

$$
F[f](\omega):=\int_{\mathbb{R}} f(x) e^{-i \omega x} d x=: \hat{f}(\omega) .
$$

Remark 2.3. Note that the Fourier transform (CFT or DDFT) can be extended to compactly supported functions (distributions) from the space $S^{\prime}(\mathbb{R})$ (for DDFT, the space is $\left.S^{\prime}([0,2 \pi))\right)$. Note also that the Fourier transform of compactly supported, in particular, functions can be continued analytically to the whole complex plane $\mathbb{C}$.

The (continuum) convolution of two functions $f, g$ is defined as

$$
(f * g)(x):=\int_{R} f(y) g(x-y) d y .
$$

For the CFT, an analog of formula (2.2) is valid also

$$
F[f * g](\omega)=\hat{f}(\omega) \hat{g}(\omega) .
$$

\subsection{Known relations}

In this subsection, we recall some well-known and introduced by Unser and Blue [7, 8] relations.

Using the Taylor series for a function $f(z):=(1+z)^{\alpha}, z \in \mathbb{C}$, we have a binomial series for any (fractional, irrational, complex) exponent $\alpha$

$$
(1+z)^{\alpha}=\sum_{k \geq 0}\left(\begin{array}{l}
\alpha \\
k
\end{array}\right) z^{k} .
$$

Recall that the binomial coefficients, in the most general case, are defined as

$$
\left(\begin{array}{c}
m \\
n
\end{array}\right):=\frac{\Gamma(m+1)}{\Gamma(n+1) \Gamma(m-n+1)} .
$$

By the relation

$$
\left(\begin{array}{c}
-\alpha-1 \\
k
\end{array}\right)=(-1)^{k}\left(\begin{array}{c}
k+\alpha \\
k
\end{array}\right),
$$


the binomial series for a negative exponent is of the form

$$
(1+z)^{-\alpha-1}=\sum_{k \geq 0}(-1)^{k}\left(\begin{array}{c}
k+\alpha \\
k
\end{array}\right) z^{k} .
$$

The binomial series for the symmetric case is, see [8],

$$
|1+z|^{\alpha}=\sum_{k \in \mathbb{Z}}\left(\begin{array}{c}
\alpha \\
k+\frac{a}{2}
\end{array}\right) z^{k}
$$

where the binomial coefficients

$$
\left(\begin{array}{c}
\alpha \\
k+\frac{a}{2}
\end{array}\right)
$$

are even with respect to $k$.

Remark 2.4. In the case of an even negative $\alpha$, binomial coefficients (2.6) vanish if $k \leq 0$, i. e., the binomial coefficients are not even with respect to $k$, and we have to redefine the coefficients as

$$
\left(\begin{array}{c}
\alpha \\
|k|+\frac{a}{2}
\end{array}\right), \quad \alpha \in 2 \mathbb{Z}, \alpha<0, k \in \mathbb{Z} .
$$

On the other hand, for an odd negative $\alpha$ and any $k$, binomial coefficients (2.6) become infinite

$$
\left(\begin{array}{c}
\alpha \\
k+\frac{a}{2}
\end{array}\right)= \pm \infty, \quad \alpha \in 2 \mathbb{Z}+1, \alpha<0, k \in \mathbb{Z} .
$$

Remark 2.5. Note, in the fractional case of $\alpha$, the binomial series in (2.3), (2.4), and (2.5) (including any odd and excluding even $\alpha$ ) are infinite; and note also that, for $|z|<1$ or for $\Re(\alpha)>0,|z|=1$, the series converge absolutely (series (2.5) converges only on the unit circle, excluding $z=-1$ ).

The CFT of the causal monomials $x_{+}^{\alpha}$ is of the form [3]

$$
F\left[x_{+}^{\alpha}\right](\omega)= \begin{cases}(i \omega)^{-\alpha-1}, & \alpha \notin \mathbb{Z}, \alpha>-1, \\ (i \omega)^{-\alpha-1}+C \delta^{(\alpha)}, & \alpha \in \mathbb{Z}_{\geq 0} ;\end{cases}
$$

where $C$ is a constant factor, and CFT of the symmetric monomials $x_{*}^{\alpha}$ is $[3$ ]

$$
F\left[x_{*}^{\alpha}\right](\omega)=|\omega|^{-\alpha-1}, \quad \alpha \in \mathbb{R}, \alpha>-1 .
$$

Remark 2.6. In the paper, formulas like (2.8), (2.9) are accurate within some constant factors.

By the CFT, we shall use the simplest definition of the fractional derivative $D^{\alpha}$ :

$$
D^{\alpha} f(x):=F^{-1}\left[(i \omega)^{\alpha} \hat{f}(\omega)\right](x) .
$$

In the symmetric case, the (fractional, in general) derivative $D_{*}^{\alpha}$ is defined as follows

$$
D_{*}^{\alpha} f(x):=F^{-1}\left[|\omega|^{\alpha} \hat{f}(\omega)\right](x) .
$$




\section{Causal and symmetric B-splines}

In the next subsections, we consider the causal and symmetric (fractional and integer) cardinal B-splines. And by a symbol $B^{\alpha}$ we shall denote B-splines of (fractional, in general) order $\alpha$ independently of their support and symmetry.

Remark 3.1. In the paper, similarly to the Fourier transform of the causal and symmetric monomials, see Remark 2.6. we define causal and symmetric B-splines within some constant factors, i. e., we use no prefactors to define the splines.

\subsection{Causal splines}

The causal B-splines $B_{+}^{\alpha}, \alpha \in \mathbb{R}, \alpha>-1$, are defined as follows

$$
B_{+}^{\alpha}(x):=\Delta_{+}^{\alpha+1} x_{+}^{\alpha}=\sum_{k \geq 0}(-1)^{k}\left(\begin{array}{c}
\alpha+1 \\
k
\end{array}\right)(x-k)_{+}^{\alpha},
$$

where $\Delta_{+}^{\alpha}$ is a forward finite difference:

$$
\Delta_{+}^{\alpha} f(x):=\sum_{k \geq 0}(-1)^{k}\left(\begin{array}{l}
\alpha \\
k
\end{array}\right) f(x-k) .
$$

Recall that the classical B-splines satisfy two-scale relations. In other words, any Bspline is a scaling function in the corresponding multiresolution analysis (MRA). For causal B-splines, the situation is the same. Let the sequence $\boldsymbol{a}:=\left(a_{k}\right)_{k \in \mathbb{Z}_{\geq 0}}$ define the mask as

$$
\hat{\boldsymbol{a}}(\omega):=\mathfrak{F}[\boldsymbol{a}](\omega)=\sum_{k=0}^{\infty} a_{k} e^{-i k \omega} .
$$

Suppose mask (3.3) has a zero of (fractional) multiplicity $\alpha+1$ at the point $\pi$. Suppose also $\hat{\boldsymbol{a}}(0)=1$, then the mask $\hat{\boldsymbol{a}}$ is of the form

$$
\hat{\boldsymbol{a}}(\omega)=\left(\frac{1+e^{-i \omega}}{2}\right)^{\alpha+1}
$$

and, using binomial expansion (2.3), we have

$$
a_{k}:= \begin{cases}\frac{1}{2^{\alpha+1}}\left(\begin{array}{c}
\alpha+1 \\
k
\end{array}\right), & k \geq 0 \\
0, & k<0 .\end{cases}
$$

By mask (3.4) and similarly to the known formula for $\cos (\omega): \prod_{j=1}^{\infty} \cos \left(2^{-j} \omega\right)=\frac{\sin \omega}{\omega}$, see, for example, the book [1, we can easily determine $\hat{B}_{+}^{\alpha}$. Namely, we have

$$
\hat{B}_{+}^{\alpha}(\omega)=\left(\frac{1-e^{-i \omega}}{i \omega}\right)^{\alpha+1}
$$

Note that the series in (3.1) is a binomial expansion of the numerator in the right-hand side of (3.5); consequently all fractional B-splines (3.1) cannot be compactly supported (in fact, $\left.\operatorname{supp} B_{+}^{\alpha}=[0, \infty)\right)$.

Using forward finite difference (3.2), formula (3.5), and fractional derivative definition (2.10), we can obtain the following relation

$$
D^{\beta} B_{+}^{\alpha}=\Delta_{+}^{\beta} B_{+}^{\alpha-\beta},
$$

which is valid for any $\alpha, \beta \in \mathbb{R}, \alpha>-1, \beta<\alpha+1$. 


\subsection{Symmetric splines}

The symmetric (fractional) B-splines $B_{*}^{\alpha}, \alpha \in \mathbb{R}, \alpha>-1$, are defined as

$$
B_{*}^{\alpha}(x):=\Delta_{*}^{\alpha+1} x_{*}^{\alpha}=\sum_{k \in \mathbb{Z}}(-1)^{k}\left(\begin{array}{c}
\alpha+1 \\
k+\frac{\alpha+1}{2}
\end{array}\right)(x-k)_{*}^{\alpha},
$$

where the symmetric monomial $x_{*}$ is defined by $(1.2)$ and $\Delta_{*}^{\alpha+1}$ is a symmetric finite difference

$$
\Delta_{*}^{\alpha} f(x):=\sum_{k \in \mathbb{Z}}(-1)^{k}\left(\begin{array}{c}
\alpha \\
k+\frac{\alpha}{2}
\end{array}\right) f(x-k) .
$$

Similarly to (3.5), the Fourier transform of the symmetric B-spline can be determined as

$$
\hat{B}_{*}^{\alpha}(\omega)=\left|\frac{1-e^{-i \omega}}{\omega}\right|^{\alpha+1} .
$$

Naturally, any symmetric B-spline satisfies a two-scale relation and the DDFT of the corresponding sequence $\boldsymbol{a}_{*}:=\left(a_{*, k}\right)_{k \in \mathbb{Z}}$, which must be even: $\hat{\boldsymbol{a}}_{*}(-\omega)=\hat{\boldsymbol{a}}_{*}(\omega)$, is of the form

$$
\hat{\boldsymbol{a}}_{*}(\omega)=\left|\frac{1+e^{-i \omega}}{2}\right|^{\alpha+1}, \quad \text { where } \quad a_{*, k}:=\frac{1}{2^{\alpha+1}}\left(\begin{array}{c}
\alpha+1 \\
k+\frac{\alpha+1}{2}
\end{array}\right), \quad k \in \mathbb{Z} .
$$

Note obvious fact that the support of any symmetric spline $B_{*}^{\alpha}$, excluding any odd order $\alpha$, is $\mathbb{R}$.

Now, present an analog of formula (3.6), see [7,

$$
D_{*}^{\beta} B_{*}^{\alpha}=\Delta_{*}^{\beta} B_{*}^{\alpha-\beta},
$$

where $\Delta_{*}^{\beta}$ is finite difference (3.8) and the derivative $D_{*}^{\beta}$ is defined by (2.11).

Finally present a convolution relation, which is valid for (classical, causal, symmetric) B-splines of any order,

$$
B^{\alpha_{1}} * B^{\alpha_{2}}=B^{\alpha_{1}+\alpha_{2}+1} .
$$

\subsection{Classical B-splines}

Classical cardinal B-splines, i. e., the splines defined by (1.1), can be considered as causal or symmetric. In fact, a classical B-spline $B^{n}$ of some order $n \in \mathbb{Z}_{\geq 0}$ is causal if it is allocated so that $\operatorname{supp} B^{n}=[0, n+1)$. A classical B-spline of some odd (positive) order is symmetric if $\operatorname{supp} B^{n}=[-\lceil n / 2\rceil,\lceil n / 2\rceil], n \in 2 \mathbb{Z}_{\geq 0}+1$.

\section{Reproducing monomials}

\subsection{Ordinary polynomials}

A fractional spline $B^{\alpha}$ satisfies the Strang-Fix conditions of order $\lceil\alpha\rceil$. Namely,

$$
\hat{B}^{\alpha}(0)=1, \quad\left(\hat{B}^{\alpha}\right)^{(m)}(2 \pi k), \quad m=0, \ldots,\lceil\alpha\rceil, k \in \mathbb{Z} \backslash\{0\} .
$$

Note that, unlike the traditional Strang-Fix theory, when compactly supported basis functions are considered and the question about convergence of reproducing does not arise; in the case of fractional splines, we have infinite series and we must trouble ourselves the convergence problem. 
In paper [8], there is a plot, see Figure 4.1 there, where, in accordance with the StrangFix conditions, a linear polynomial is reproduced by integer shifts of the spline $B_{+}^{\frac{1}{2}}$ :

$$
\sum_{k \in \mathbb{Z}}\left(k+\frac{3}{4}\right) B_{+}^{\frac{1}{2}}(x-k)=x, \quad x \in \mathbb{R} .
$$

Nevertheless, this reproducing demonstrates a problem with the series in the left-hand side of (4.1). Namely, considering equality (4.1) on the ray $[0, \infty)$, applying derivative $D^{\frac{1}{2}}$ to the both sides of the equality, and using (3.6), we get

$$
x \in[0, \infty): \quad \sum_{k \in \mathbb{Z}}\left(k+\frac{3}{4}\right) \Delta_{+}^{\frac{1}{2}} B_{+}^{0}(x-k)=\sqrt{x},
$$

where $B_{+}^{0}=B^{0}$, in fact, is the indicator function of the interval $[0,1)$. Since the sum of the series in the left-hand side of (4.2) is a step function, it follows that this equality cannot take place. Thus we have to suppose that the series in the left-hand side of (4.1) does not converge uniformly (only the pointwise convergence is valid); consequently, the series cannot be differentiated (the Fourier transform cannot be applied) component-wise. Moreover, we can demonstrate that the partition of the unity by $B_{+}^{\frac{1}{2}}$ also converges nonuniformly. Namely, similarly to the previous case, we have an impossible situation

$$
x \in[0, \infty): \quad \sum_{k \in \mathbb{Z}} \Delta_{+}^{\frac{1}{2}} B^{0}(x-k)=\frac{1}{\sqrt{x}} .
$$

Here we do not investigate explicitly convergence of the considered series.

\subsection{Causal and symmetric monomials}

As has been demonstrated, fractional causal and symmetric B-splines are formed by linear combinations of shifted monomials, see (3.1), (3.7). So we can expect to obtain the causal and symmetric fractional monomials by the corresponding causal and symmetric fractional B-splines.

\subsubsection{Causal monomials}

Let $\hat{\boldsymbol{a}}(\omega):=\mathfrak{F}[\boldsymbol{a}](\omega), \boldsymbol{a}:=\left(a_{k}\right)_{k \in \mathbb{Z}_{\geq 0}}$, be the mask that defines the scaling function (B-spline $\left.B_{+}^{\alpha}\right)$ and be of the form (3.4). Let a detailed mask $\hat{\boldsymbol{b}}(\omega):=\mathfrak{F}[\boldsymbol{b}](\omega), \boldsymbol{b}:=\left(b_{k}\right)_{k \in \mathbb{Z}_{\geq 0}}$, which defines a wavelet, be determined as follows

$$
\hat{\boldsymbol{b}}(\omega):=\hat{\boldsymbol{a}}(\omega+\pi)=\left(\frac{1-e^{-i \omega}}{2}\right)^{\alpha+1} .
$$

And, consequently, the elements of $\boldsymbol{b}$ are

$$
b_{k}:=(-1)^{k} a_{k}= \begin{cases}(-1)^{k} \frac{1}{2^{\alpha+1}}\left(\begin{array}{c}
\alpha+1 \\
k
\end{array}\right), & k \geq 0 ; \\
0, & k<0 .\end{cases}
$$

Theorem 4.1. Let $B_{+}^{\alpha}$ be a causal spline of order $\alpha$, where $\alpha \in \mathbb{R}, \alpha>-1$, is an arbitrary number. Suppose a sequence $p$ is

$$
\boldsymbol{p}=\left(p_{k}\right)_{k \in \mathbb{Z}_{\geq 0}}, \quad p_{k}:= \begin{cases}\left(\begin{array}{c}
k+\alpha \\
k
\end{array}\right), & k \in \mathbb{Z}_{\geq 0}, \\
0, & k<0\end{cases}
$$


then we have

$$
\sum_{k=0}^{\infty} p_{k} B_{+}^{\alpha}(x-k)=\sum_{k=0}^{\infty}\left(\begin{array}{c}
k+\alpha \\
k
\end{array}\right) B_{+}^{\alpha}(x-k)=x_{+}^{\alpha} .
$$

Proof. Consider two cases.

$\alpha \notin \mathbb{Z}$. Using binomial series (2.4), the DDFT of sequence (4.4) can be written as follows

$$
\hat{\boldsymbol{p}}(\omega):=\sum_{k=0}^{\infty}\left(\begin{array}{c}
k+\alpha \\
k
\end{array}\right) e^{-i k \omega}=\left(\frac{1}{1-e^{-i \omega}}\right)^{\alpha+1}
$$

Applying the Fourier transform to the both sides of (4.5), we obtain

$$
\sum_{k=0}^{\infty} p_{k} e^{-i k \omega} \hat{B}_{+}^{\alpha}(\omega)=(i \omega)^{-\alpha-1}
$$

Using (3.5) and (4.6), we get

$$
\frac{1}{\left(1-e^{-i \omega}\right)^{\alpha+1}}\left(\frac{1-e^{-i \omega}}{i \omega}\right)^{\alpha+1}=\left(\frac{1}{i \omega}\right)^{\alpha+1} .
$$

Thus equality (4.5) is valid.

$\alpha \in \mathbb{Z}$. Since the expression $\left(1-e^{-i \omega}\right)^{\alpha+1}$ has a zero of multiplicity $\alpha+1$ at the point 0 ; therefore, we can rewrite the Fourier transform of the spline $B_{+}^{\alpha}$, see (3.5), as follows

$$
\begin{aligned}
\hat{B}_{+}^{\alpha}(\omega)=\left(\frac{1-e^{-i \omega}}{i \omega}\right)^{\alpha+1} & +C \delta^{(\alpha)}\left(1-e^{-i \omega}\right)^{\alpha+1} \\
& =\left(1-e^{-i \omega}\right)^{\alpha+1}\left((i \omega)^{-\alpha-1}+C \delta^{(\alpha)}\right)
\end{aligned}
$$

where $C$ is a constant factor. Using (2.8) (the second line) and (4.6), we obtain (4.5). This completes the proof.

In Fig. 1, we present reproducing of the causal monomials $x_{+}^{\alpha}, \alpha=\frac{1}{5}, \frac{3}{4}, 1, \frac{5}{4}$, by a linear combination of integer shifts of the causal B-splines $B_{+}^{\alpha}$, see (4.5).

Remark 4.1. Sometimes, this is more convenient to rewrite formula (4.5) as follows

$$
x_{+}^{\alpha}=\Delta_{+}^{-\alpha-1} B_{+}^{\alpha},
$$

where the causal finite difference is defined by (3.2).

\subsubsection{Symmetric monomials}

Let $\hat{\boldsymbol{a}}_{*}(\omega):=\mathfrak{F}\left[\boldsymbol{a}_{*}\right](\omega), \boldsymbol{a}_{*}:=\left(a_{*, k}\right)_{k \in \mathbb{Z}}$, be a mask. Let the mask $\hat{\boldsymbol{a}}_{*}$ be of the form (3.10), i. e., the mask has a zero of multiplicity $\alpha+1$ at the point $\pi$ and $\hat{\boldsymbol{a}}_{*}(0)=1$. Consequently, the mask can be used to define the B-spline $B_{*}^{\alpha}$. Let a detailed mask $\hat{\boldsymbol{b}}$ be determined as follows

$$
\hat{\boldsymbol{b}}(\omega):=\hat{\boldsymbol{a}_{*}}(\omega+\pi)=\left|\frac{1-e^{-i \omega}}{2}\right|^{\alpha+1} .
$$

And, consequently, the terms of $\boldsymbol{b}$ are

$$
b_{k}=(-1)^{k} a_{k}=(-1)^{k} \frac{1}{2^{\alpha+1}}\left(\begin{array}{c}
\alpha+1 \\
k+\frac{\alpha+1}{2}
\end{array}\right), \quad k \in \mathbb{Z} .
$$




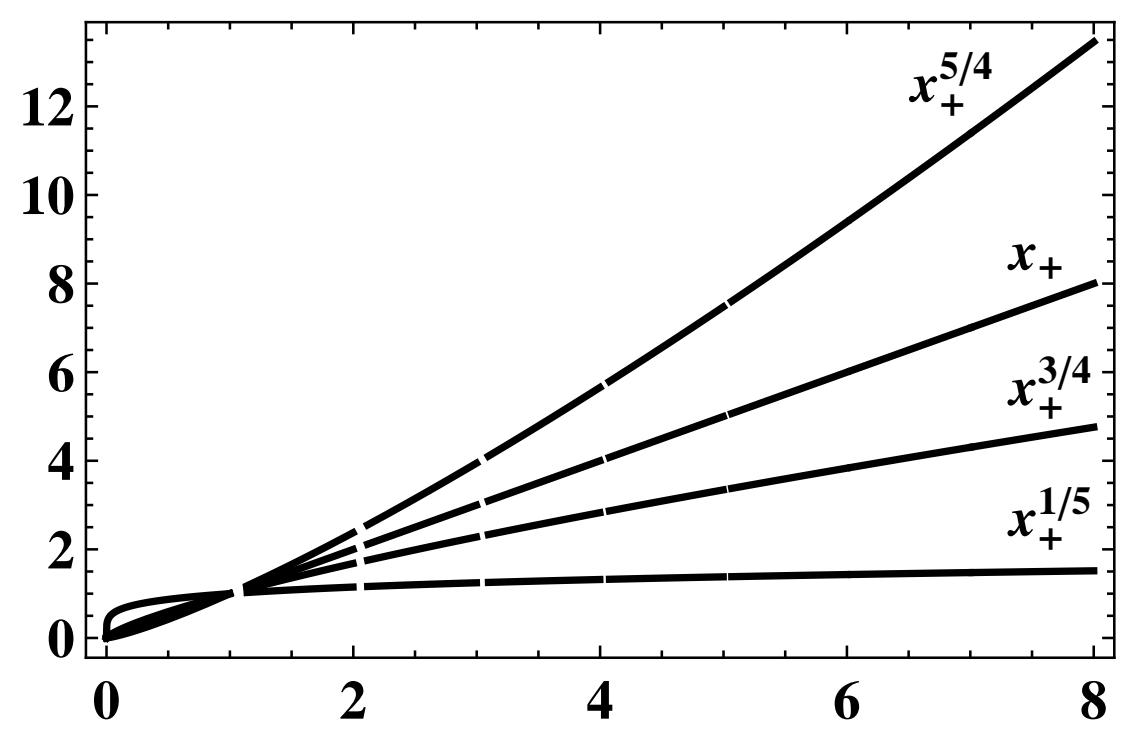

Figure 1: Reproducing of the causal monomials $x_{+}^{\alpha}, x \in[0, \infty), \alpha=\frac{1}{5}, \frac{3}{4}, 1, \frac{5}{4}$, by the causal B-splines $B_{+}^{\alpha}$.

Theorem 4.2. Let $B_{*}^{\alpha}$ be a symmetric spline of order $\alpha$, where $\alpha \in \mathbb{R}, \alpha>-1$, is an arbitrary number. Suppose a sequence $\boldsymbol{p}$ is

$$
\boldsymbol{p}=\left(p_{k}\right)_{k \in \mathbb{Z}}, \quad p_{k}:=(-1)^{k}\left(\begin{array}{c}
-\alpha-1 \\
k-\frac{\alpha+1}{2}
\end{array}\right), \quad k \in \mathbb{Z},
$$

then

$$
\sum_{k \in \mathbb{Z}} p_{k} B_{*}^{\alpha}(x-k)=\sum_{k \in \mathbb{Z}}(-1)^{k}\left(\begin{array}{c}
-\alpha-1 \\
k-\frac{\alpha+1}{2}
\end{array}\right) B_{*}^{\alpha}(x-k)=x_{*}^{\alpha},
$$

where $x_{*}^{\alpha}$ is defined by (1.2).

The proof of this theorem is similar to the proof of Theorem 4.1 and based on formulas (2.5), (3.9).

In Fig. 2, we present reproducing of the symmetric monomials $x_{*}^{\alpha}, \alpha=\frac{1}{2}, 1, \frac{3}{2}, 2$, by integer shifts of the symmetric B-splines $B_{*}^{\alpha}$, see (4.10).

Remark 4.2. In spite of the infinite support of a symmetric B-spline $B_{*}^{\alpha}, \alpha \notin 2 \mathbb{Z}_{\geq 0}+1$, the series in the left-hand side of formula (4.10) converges uniformly; and we can apply component-wise the fractional derivative $D_{*}$ of some order $<\alpha+1$ to the both sides of relation (4.10). Unlike reproducing of ordinary polynomials by the fractional B-splines, formula (4.10) can be considered as an analytical manipulation of the shifted B-splines to obtain (fractional) monomials on the whole line $\mathbb{R}$ at once.

In the symmetric case, the similar to (4.7) formula is valid also

$$
x_{*}^{\alpha}=\Delta_{*}^{-\alpha-1} B_{*}^{\alpha} .
$$

\subsubsection{Causal and symmetric monomials of integral degree}

As has been noted in Subsection 3.3, the classical B-splines can be considered as particular cases of the causal and symmetric B-splines. Thus formulas (4.5) and (4.10) can be applied to the classical B-splines, see Figs. 1, 2. 


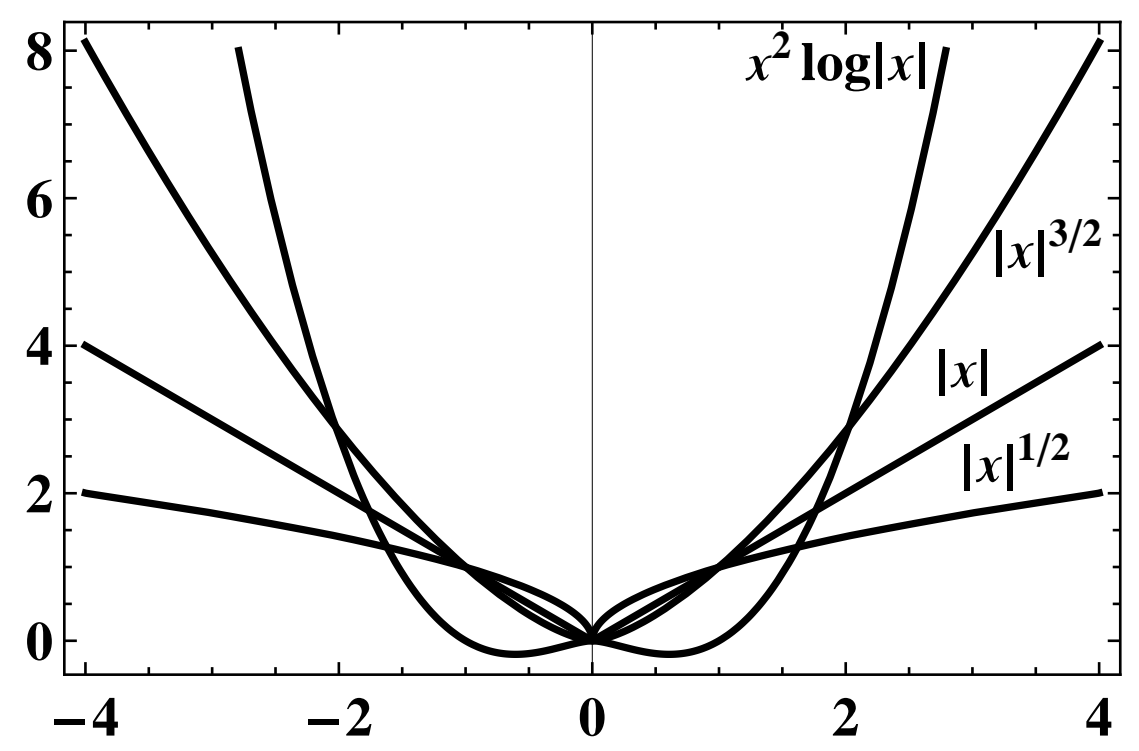

Figure 2: Reproducing of symmetric monomials $x_{*}^{\alpha}, x \in \mathbb{R}, \alpha=\frac{1}{2}, 1, \frac{3}{2}, 2$, by the symmetric B-splines $B_{*}^{\alpha}$.

According to Remark 2.4, for any even degree $\alpha$ of the reproduced monomial $x_{*}^{\alpha}, \alpha \in$ $2 \mathbb{Z}_{\geq 0}$, formula (4.10) cannot be applicable. However this is possible to factorize a finite difference $\Delta_{*}^{\alpha}$ as follows

$$
\Delta_{*}^{\alpha}=\Delta_{*}^{\alpha_{1}} * \Delta_{*}^{\alpha_{2}} * \cdots * \Delta_{*}^{\alpha_{k}}, \quad \begin{aligned}
& \alpha_{1}+\alpha_{2}+\cdots+\alpha_{k}=\alpha, \\
& a_{j} \neq-2 \mathbb{N}+1, j=1, \ldots, k,
\end{aligned}
$$

where orders $\alpha_{j}, j=1, \ldots k$, are arbitrary (excluding negative odd values).

Using (4.11) and decomposition $3=\frac{3}{2}+\frac{3}{2}$, for example, the monomial $x_{*}^{2}:=x^{2} \log |x|$ can be reproduced as

$$
\begin{aligned}
x^{2} \log |x|=\Delta_{*}^{-3} B_{*}^{2}(x)=\left(\Delta_{*}^{-3 / 2} * \Delta_{*}^{-3 / 2}\right) & B_{*}^{2}(x) \\
& =\Delta_{*}^{-3 / 2}\left(\Delta_{*}^{-3 / 2} B_{*}^{2}(x)\right) .
\end{aligned}
$$

Note that the series in (4.12) converge very slowly and this technique can be interesting from the methodical point of view only.

\subsection{Two-dimensional case}

In this subsection, we consider the simplest two-dimensional cases of the causal and symmetric B-splines (fractional and integer) obtained by tensor product of the one-dimensional B-splines. Namely,

$$
\begin{aligned}
& B_{+}^{\alpha_{1}, \alpha_{2}}(x, y):=B_{+}^{\alpha_{1}}(x) B_{+}^{\alpha_{2}}(y) \\
& \quad=\sum_{k_{1}, k_{2} \geq 0}(-1)^{k_{1}+k_{2}}\left(\begin{array}{c}
\alpha_{1}+1 \\
k_{1}
\end{array}\right)\left(\begin{array}{c}
\alpha_{2}+1 \\
k_{2}
\end{array}\right)\left(x-k_{1}\right)_{+}^{\alpha_{1}}\left(y-k_{2}\right)_{+}^{\alpha_{2}}, \quad x \geq 0, y \geq 0 ; \\
& B_{*}^{\alpha_{1}, \alpha_{2}}(x, y):=B_{*}^{\alpha_{1}}(x) B_{*}^{\alpha_{2}}(y) \\
& \quad=\sum_{k_{1}, k_{2} \in \mathbb{Z}}(-1)^{k_{1}+k_{2}}\left(\begin{array}{c}
\alpha_{1}+1 \\
k_{1}+\frac{\alpha_{1}+1}{2}
\end{array}\right)\left(\begin{array}{c}
\alpha_{2}+1 \\
k_{2}+\frac{\alpha_{2}+1}{2}
\end{array}\right)\left(x-k_{1}\right)_{*}^{\alpha_{1}}\left(y-k_{2}\right)_{*}^{\alpha_{2}}, \quad x, y \in \mathbb{R} .
\end{aligned}
$$


Note that the support of any two-dimensional causal B-spline $B_{+}^{\alpha_{1}, \alpha_{2}}(x, y)$ (if $\alpha_{1}, \alpha_{2}$ are fractional) is the first quadrant of the Cartesian plane $\mathbb{R}^{2}$ and the support of any symmetric B-spline $B_{*}^{\alpha_{1}, \alpha_{2}}(x, y)$ (if $\alpha_{1}, \alpha_{2}$ are not odd integer) is the whole plane $\mathbb{R}^{2}$.

The Fourier transform of two-dimensional B-splines obviously is

$$
\begin{aligned}
& \hat{B}_{+}^{\alpha_{1}, \alpha_{2}}(\xi, \eta):=\hat{B}_{+}^{\alpha_{1}}(\xi) \hat{B}_{+}^{\alpha_{2}}(\eta)=\left(\frac{1-e^{-i \xi}}{i \xi}\right)^{\alpha_{1}+1}\left(\frac{1-e^{-i \eta}}{i \eta}\right)^{\alpha_{2}+1}, \\
& \hat{B}_{*}^{\alpha_{1}, \alpha_{2}}(\xi, \eta):=\hat{B}_{*}^{\alpha_{1}}(\xi) \hat{B}_{*}^{\alpha_{2}}(\eta)=\left|\frac{1-e^{-i \xi}}{\xi}\right|^{\alpha_{1}+1}\left|\frac{1-e^{-\eta}}{\eta}\right|^{\alpha_{2}+1} .
\end{aligned}
$$

Having some B-spline $B_{+}^{\alpha_{1}, \alpha_{2}}$, or $B_{*}^{\alpha_{1}, \alpha_{2}}$, we can obtain a two-dimensional monomial $x_{+}^{\alpha_{1}} y_{+}^{\alpha_{2}}$, or $x_{*}^{\alpha_{1}} y_{*}^{\alpha_{2}}$, respectively, as follows

$$
\begin{aligned}
& \sum_{k_{1}, k_{2} \geq 0}\left(\begin{array}{c}
k_{1}+\alpha_{1} \\
k_{1}
\end{array}\right)\left(\begin{array}{c}
k_{2}+\alpha_{2} \\
k_{2}
\end{array}\right) B_{+}^{\alpha_{1}, \alpha_{2}}\left(x-k_{1}, y-k_{2}\right)=x_{+}^{\alpha_{1}} y_{+}^{\alpha_{2}} \\
& \sum_{k_{1}, k_{2} \in \mathbb{Z}}(-1)^{k_{1}+k_{2}}\left(\begin{array}{c}
-\alpha_{1}-1 \\
k_{1}-\frac{\alpha_{1}+1}{2}
\end{array}\right)\left(\begin{array}{c}
-\alpha_{2}-1 \\
k_{2}-\frac{\alpha_{2}+1}{2}
\end{array}\right) B_{*}^{\alpha_{1}, \alpha_{2}}\left(x-k_{1}, y-k_{2}\right)=x_{*}^{\alpha_{1}} y_{*}^{\alpha_{2}} .
\end{aligned}
$$

Similarly to the one-dimensional case, two-dimensional causal monomials are reproduced exactly (on any bounded set of the first quadrant) by a finite number of the bivariate causal B-splines.

Modifying a little summation in formula (4.14), we can obtain that the support of the reproduced symmetric monomial $x_{*}^{\alpha_{1}} y_{*}^{\alpha_{2}}$ is the first and third quadrants (or the second and fourth quadrants) of the plane $\mathbb{R}^{2}$. Namely, the indexes of summation $k_{1}, k_{2}$ in the left-hand side of formula (4.14) must have the same (or opposite, in the second case) signs; i. e., $k_{1} k_{2} \geq 0$ (or $k_{1} k_{2} \leq 0$, respectively).

In Fig. 3, we present reproducing of 2D monomials (causal and symmetric) by integer shifts of the corresponding 2D B-splines, see (4.13), (4.14).
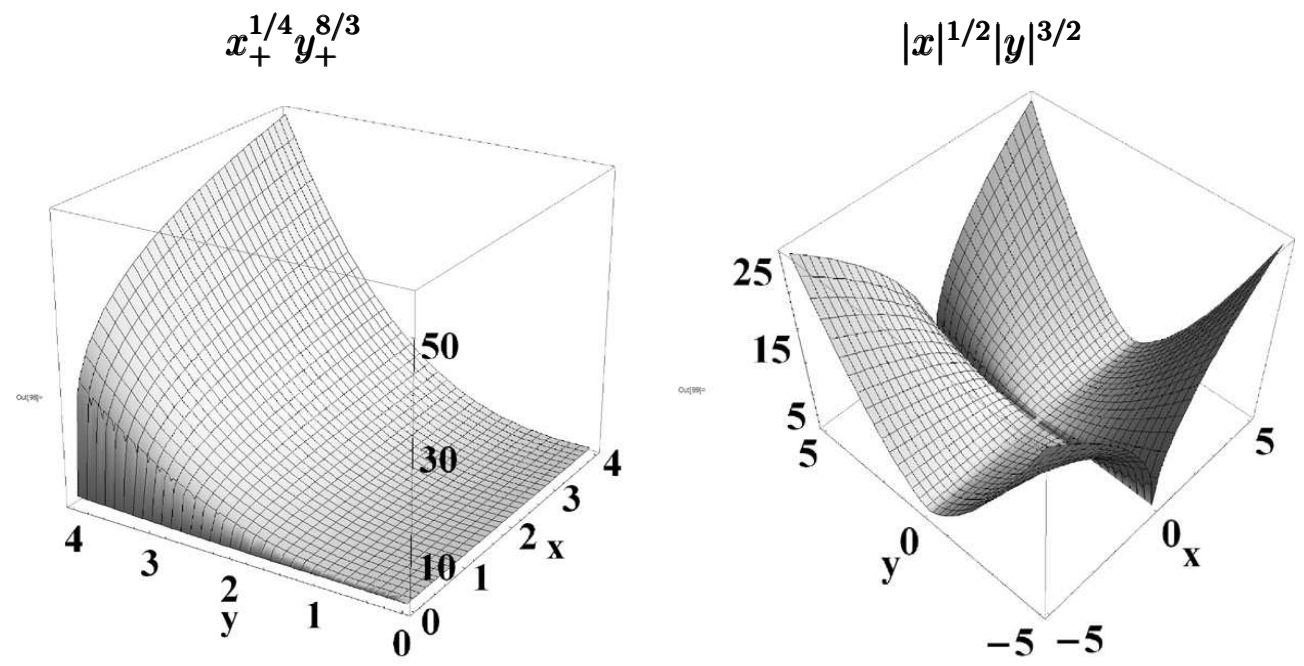

Figure 3: 2D causal monomial $x_{+}^{1 / 4} y_{+}^{8 / 3}, x, y \in \mathbb{R}^{2}, x, y \geq 0$, reproduced by the $2 \mathrm{D}$ causal Bspline $B_{+}^{1 / 4,8 / 3}(x, y)$ (left); and $2 \mathrm{D}$ symmetric monomial $|x|^{1 / 2}|y|^{3 / 2}, x, y \in \mathbb{R}^{2}$, reproduced by the $2 \mathrm{D}$ symmetric B-spline $B_{*}^{1 / 2,3 / 2}(x, y)$ (right). 


\section{The Strang-Fix conditions}

In this section, we considered reproducing of the causal and symmetric monomials (integer and fractional) with relation to the Strang-Fix conditions. The Strang-Fix conditions are presented in the context of numerical sequences (filters) and MRA. This section can be interpreted as an announcement and the subdivision approach to the Strang-Fix theory will be the object of other papers, see paper $[9$.

\subsection{Classical Strang-Fix conditions}

Theorem 5.1. Let $\boldsymbol{a}=\left(a_{k}\right)_{k \in \mathbb{Z}}$ be a sequence and let $\boldsymbol{b}=\left(b_{k}\right)_{k \in \mathbb{Z}}$ be another sequence defined as

$$
\boldsymbol{b}:=\mathfrak{F}^{-1} \hat{\boldsymbol{a}}(\omega+\pi)
$$

Suppose $\hat{\boldsymbol{a}}(0)=1$ and

$$
\left|\begin{array}{ll}
\hat{\boldsymbol{a}}(\omega) & \hat{\boldsymbol{a}}(\omega+\pi) \\
\hat{\boldsymbol{b}}(\omega) & \hat{\boldsymbol{b}}(\omega+\pi)
\end{array}\right| \neq 0, \quad \omega \in[0,2 \pi)
$$

Let $p(x), x \in \mathbb{R}$, be a function and $\boldsymbol{p}=(p(k))_{k \in \mathbb{Z}}$ be samples of the function $p$ (selected in the unit step of discretization). Let $\phi$ be the scaling function corresponding to the sequence $\boldsymbol{a}: \hat{\phi}(\omega):=\prod_{j=1}^{\infty} \hat{\boldsymbol{a}}\left(\omega / 2^{j}\right)$. Suppose

$$
\boldsymbol{b} * \boldsymbol{p}=0
$$

then reproducing relations

$$
\sum_{k \in \mathbb{Z}} p(k) \phi(x-k)=\sum_{k \in \mathbb{Z}} \phi(k) p(x-k)=p * \phi(x) .
$$

are valid.

Remark 5.1. Here note some remarks on the previous theorem.

1. In fact, conditions (5.3) are equivalent to the traditional Strang-Fix conditions on the basis function $\phi$ (defined by $\boldsymbol{a}$ ) and the reproduced function p. Certainly, conditions (5.3) do not supply directly the order of the corresponding Strang-Fix conditions. The order is defined by the sequence $\boldsymbol{b}$, actually, by the corresponding sequence $\boldsymbol{a}$ (scaling function $\phi$ ).

2. Definition of the sequence $\boldsymbol{b}$ by formula (5.1) is not necessary and, consequently, $\boldsymbol{b}$ can be defined by a different way. In any case, the sequences $\boldsymbol{a}$ and $\boldsymbol{b}$ must provide (5.2).

3. Condition (5.2) is valid iff the following sum that defines the corresponding MRA

$$
V_{1}=V_{0} \oplus W_{0} \quad\left(\begin{array}{l}
\text { the direct sum is not } \\
\text { necessary orthogonal }
\end{array}\right),
$$

where $V_{0}:=\operatorname{span}\{\phi(\cdot-k): k \in \mathbb{Z}\}, V_{1}:=\operatorname{span}\{\sqrt{2} \phi(2 \cdot-k): k \in \mathbb{Z}\}, \quad \psi:=\boldsymbol{b}$. $(\sqrt{2} \phi(2 \cdot-k))_{k \in \mathbb{Z}}$, and $W_{0}:=\operatorname{span}\{\psi(\cdot-k): k \in \mathbb{Z}\}$, holds.

4. If we rescale the function $p$ as: $p^{\sharp}:=p(c \cdot)$, where $c$ is a rescaling factor; then, for the function $p^{\sharp}$, conditions (5.3) are valid also. Thus $p$ can be sampled in an arbitrary step. 
Using the DDFT, rewrite conditions (5.3) as:

$$
\hat{\boldsymbol{b}}(\omega) \hat{\boldsymbol{p}}(\omega)=0, \quad \omega \in[0,2 \pi) .
$$

Let the sequence $\boldsymbol{a}$ (hence, the sequence $\mathbf{b}$ also) is compactly supported, then $\hat{\boldsymbol{b}}(\omega)$ can vanish on a set of zero measure only. Consequently, condition (5.4) is valid iff the function $\hat{\boldsymbol{p}}(\omega)$ has a zero support. There is a fact that any distribution that is concentrated at one point is a finite sum of the delta-distribution and its derivatives. Thus, if the sequence $\boldsymbol{a}$ is compactly supported, the Strang-Fix conditions can take place only for algebraic polynomials. If the function $\hat{\boldsymbol{b}}(\omega)$ has a zero of multiplicity $m \in \mathbb{N}$ at the point $x_{0} \in \mathbb{R}$, then a polynomial of degree no more than $m-1$ satisfies condition (5.4).

Remark 5.2. If we continue analytically condition (5.4) to the whole plane $\mathbb{C}$, see Remark 2.3; and if the analytical continuation of the function $\hat{\boldsymbol{b}}(\omega), \omega \in \mathbb{C}$, has a zero of multiplicity $m \in \mathbb{N}$ at a point $x_{0} \in \mathbb{C}$. Then the functions $x^{n} e^{x_{0} x}, n=0,1, \ldots, m-1$, satisfy condition (5.4) (equivalently, (5.3)) and can be reproduced by the corresponding function $\phi$. In the paper, we shall not consider this generalization. Here note only the papers [10, 11].

Moreover, note that this approach can be extended to fractional exponential splines, see paper [4]. And the monomials (multiplied by exponents) can be represented by the corresponding B-splines. This will be discussed elsewhere.

\subsection{Weakening of the Strang-Fix conditions}

The Fourier transform of a causal $x_{+}^{\alpha}$ or symmetric $x_{*}^{\alpha}$ monomial, where $\alpha \in \mathbb{R}$ is not necessary fractional, cannot comply with condition (5.4) if the function $\hat{\boldsymbol{b}}(\omega)$ vanishes only at a point set. Thus we have to weaken the Strang-Fix conditions (5.3) and formulate a weakened theorem.

Theorem 5.2. Under the conditions of Theorem 5.1, weakening the Strang-Fix conditions (5.3) as follows

$$
\boldsymbol{b} * \boldsymbol{p}=\delta_{n 0} ;
$$

we have

$$
\sum_{k \in \mathbb{Z}} p(k) \phi(x-k)=\sum_{k \in \mathbb{Z}} \phi(k) p(x-k)=p * \phi(x) .
$$

Applying the DDFT to the both sides of condition (5.5), we have

$$
\hat{\boldsymbol{b}}(\omega) \hat{\boldsymbol{p}}(\omega)=1, \quad \text { a. e. }
$$

And, using (4.3), (4.6), (4.8), (4.9), we see that, in Theorems 4.1, 4.2, condition (5.6) (equivalently, (5.5) ) holds, i. e., Theorems 4.1, 4.2 can be considered as particular cases of Theorem 5.2 ,

Remark 5.3. In Theorem 5.2, we do not specify the function $p=p(x), x \in \mathbb{R}$. This is a nontrivial problem and to define the function we need many additional explanations. Note also that even in Theorems 4.1. 4.2, we do not consider this function, only sequence.

Condition (5.5), in the causal case, can be written in a matrix form that provides with the following system of linear equations

$$
\left(\begin{array}{cccccc}
b_{0} & 0 & 0 & 0 & 0 & \ldots \\
b_{1} & b_{0} & 0 & 0 & 0 & \ldots \\
b_{2} & b_{1} & b_{0} & 0 & 0 & \ldots \\
b_{3} & b_{2} & b_{1} & b_{0} & 0 & \ldots \\
\ldots & \ldots & \ldots & \ldots & \ldots
\end{array}\right)\left(\begin{array}{c}
p_{0} \\
p_{1} \\
p_{2} \\
p_{3} \\
p_{4} \\
\vdots
\end{array}\right)=\left(\begin{array}{c}
1 \\
0 \\
0 \\
0 \\
\vdots
\end{array}\right) .
$$


Note that system of linear equations (5.7) supplies convenient matrix form of the weakened Strang-Fix conditions. Namely, we do not bother ourselves about convergence of the binomial or Taylor series. Moreover, the matrix form provides an useful way to state and solve the multidimensional weakened Strang-Fix conditions. Note also that, in the multidimensional case, several nonzero members of convolution (5.5) (on the boundary of a compactly supported sequence $\boldsymbol{a}$, for example) are natural.

This will be the object of another paper.

Remark 5.4. This is a well-known fact, that the space of polynomials up to some integral degree $m$ is invariant under an arbitrary shift; and the dimension of such space is $m+1$. So the ordinary Strang-Fix conditions provide for reproducing, by integer shifts of basis functions (B-splines, in our case), of any polynomial (of degree up to the Strang-Fix conditions order).

In the case of causal and symmetric (fractional and integer) monomials, we have onedimensional spaces, i. e., the spaces of causal and symmetric monomials are not shift invariant. Note, since the spaces of causal and symmetric monomials are reproduced by integer shifts of the corresponding B-splines; the spaces are invariant under integer shifts.

\section{References}

[1] I. Daubechies, Ten Lectures on Wavelets (SIAM, 1992).

[2] G. Fix and G. Strang, Fourier analysis of the finite element method in Ritz-Galerkin theory, Stud. Appl. Math. 48 (1969) 265-273.

[3] I.M. Gel'fand and G.E. Shilov, Generalized Functions, Vol. 1: Properties and Operations (AMS Chelsea Publishing, 2016).

[4] P. Massopust, Splines and fractional differential operators, Int. J. Wavelets Multiresolut. Inf. Process. (2020) DOI: 10.1142/S0219691320400056.

[5] I. Schoenberg, Contribution to the problem of approximation of equidistant data by analytic functions, Quart. Appl. Math. 4 (1946) pp. 45-99 and 112-141.

[6] G. Strang and G. Fix, An Analysis of the Finite Element Method (Prentice-Hall, 1973).

[7] M. Unser and T. Blu, Construction of fractional spline wavelet bases, in Proc. SPIE Conf. Wavelet Applications in Signal and Image Processing VII. (Denver CO, USA, July 19-23, 1999) Vol. 3813, pp. 422-431.

[8] M. Unser and T. Blu, Fractional Splines and Wavelets, SIAM Review, 42(1) (2000) $43-67$.

[9] V.G. Zakharov, The Strang-Fix conditions and subdivisions, In preparation.

[10] V.G. Zakharov, Reproducing solutions to PDEs by scaling functions, Int. J. Wavelets Multiresolut. Inf. Process. 3 (2020) 2050017.

[11] V.G. Zakharov, Operator-adapted wavelets: Connection with StrangFix conditions, Int. J. Wavelets Multiresolut. Inf. Process. 10 (2012) 1250006. 